\title{
The influence of hydrogen loading and the fabrication process on the mechanical strength of optical fibre Bragg gratings
}

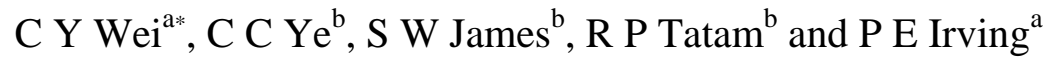 \\ a Damage Tolerance Group, School of Industrial and Manufacturing Science, \\ Cranfield University, Cranfield, Bedford MK43 0AL, UK \\ b Optical Sensors Group, Centre for Photonics and Optical Engineering, School of Engineering, \\ Cranfield University, Cranfield, Bedford MK43 0AL, UK
}

\begin{abstract}
This paper investigates the influence of hydrogen loading and other stages of the fabrication process on the mechanical strength of fibre Bragg gratings. Following UV-irradiation, tensile tests were carried out on Ge-B codoped photosensitive fibres with and without hydrogen loading. Fibre Bragg gratings (FBGs) were written using a range of UV wavelengths, namely $246 \mathrm{~nm}, 255 \mathrm{~nm}$ and 266 nm. The tensile strength of the optical fibres was determined in their as-received status and following the various stages of FBG fabrication. The mechanical strength was assessed using Weibull statistics. The results indicate that the strength of FBGs is influenced by the UV irradiation parameters and by the hydrogen-loading process. FBGs fabricated using shorter UV wavelengths and low pulse power intensity exhibit a high mechanical strength. The FBGs written in hydrogen loaded fibres have less than 50\% of the strength of FBGs that have not been hydrogen-loaded. Fibre fracture morphology observed by scanning electron microscope (SEM) reveals fracture mechanisms of FBGs, which are correlated with the structural change of the silica fibres induced during the FBG
\end{abstract}

\footnotetext{
* Correspondence author: Dr C Y Wei, Building 40, SIMS, Cranfield University, Bedford MK43 0HP, UK; Tel: +44 (0)1234 750111; Fax: +44 (0)1234 752376; E-mail:c.wei@cranfield.ac.uk
} 
inscription process. Combined with surface information gathered by atomic force microscopy (AFM), fracture mechanics is applied to understand the mechanisms of strength degradation caused by the FBG inscription process.

PACS codes: 42.50.VK; 42.79.Dj; 42.81.pa

Keywords: Fibre Bragg grating strength; Hydrogen loaded optical fibre; Bragg grating formation mechanisms, mechanical resistance of fibre Bragg gratings

\section{INTRODUCTION}

A fibre Bragg grating (FBG) consists of a periodic perturbation of the refractive index of the core of an optical fibre. FBGs are formed by exposure of pristine glass fibres to an interference pattern formed between intersecting UV laser beams. To achieve a high Bragg reflectivity, the photosensitivity of the pristine fibre is enhanced either by doping the core with elements such as Ge, or Sn [1], or by hydrogen-loading the optical fibre via immersion in high pressure hydrogen [2]. The high pressure hydrogen-loading technique has been shown to achieve ultrahigh photosensitivity in $\mathrm{GeO}_{2}$-doped fibres [3]. Hydrogen loading is commonly used to allow FBG fabrication in standard telecommunication optical fibres.

FBGs are required to have a specific degree of mechanical robustness according to their application. For instance, as sensors, the strain to failure should be higher than that of the measured object to ensure that no sensor failure occurs before the failure of the object. Previous work [4] has shown that continuous-wave (CW) UV exposure can produce high-mechanical-resistance FBGs with mean strength and m-values close to those of pristine optical fibres. However, a range of pulsed lasers are also used in the fabrication of FBGs. Gratings can be written quickly and have been fabricated from a single laser pulse during the fibre draw process [5]. It has been reported that 
the use of pulsed UV-irradiation to form FBGs degrades the mechanical strength [6, 7]. Varelas et al [7] reported that the median strength of UV irradiated fibres was around $50 \%$ of that of nonirradiated fibres. The total irradiation dose was found to have a limited influence on the mechanical strength, which varied from 2.5 to $2.7 \mathrm{GPa}$ when the total dose increased from $1.0 \mathrm{~kJ} / \mathrm{cm}^{2}$ to 3.0 $\mathrm{kJ} / \mathrm{cm}^{2}$. An investigation by Feced [8] revealed that the UV wavelength had an influence on the mechanical strength. In [8], the strengths of FBGs written using UV irradiation at 193 and $248 \mathrm{~nm}$, at an average pulse intensity of $1.0 \mathrm{~J} / \mathrm{cm}^{2}$, were 0.84 and $0.69 \mathrm{GPa}$, respectively. Following on from the work reported in [6], using laser wavelengths of 246 and 266nm, this paper examines the influence of key process parameters on the mechanical strength of irradiated fibres. These parameters include the pulse power, total dose and wavelengths of 246, 255 and 266nm.

Fabrication of FBGs while the fibre is being drawn has demonstrated that high strength FBGs can be produced [9]. No reduction in strength was observed when FBGs were written using a $\mathrm{KrF}$ excimer pulsed laser of $248 \mathrm{~nm}$ at a pulse average intensity of $1.0 \mathrm{~J} / \mathrm{cm}^{2}$ immediately after silica fibres were drawn and before the protective coating was applied to the fibres. The irradiated fibres showed a median strength of 5.8 GPa. However, on-line FBG fabrication provides low reflectivity FBGs (less than 10\%) [9]. The structural change of the irradiated fibres with low reflectivity may not reach a threshold at which mechanical damage is caused. FBGs fabricated in hydrogen-loaded fibres have a higher reflectivity than those fabricated in non-hydorgen-loaded fibres under the same irradiation conditions [3]. It is not clear whether there is a relationship between the reflectivity and the mechanical strength of FBGs. Previous research has shown no change in mechanical strength of optical fibres following hydrogen-loading [11]. However, no further work has been reported on the strength of the hydrogen-loaded fibres that are subjected subsequently to the UV-writing process. This paper determines the mechanical strength of FBGs using Ge-B codoped fibres with and without hydrogen-loading. Observation of the fracture surface, combined with the observation of 
the surface morphology using an atomic force microscopy (AFM), enables possible mechanisms for the reduction of the mechanical strength of optical fibre with different photosensitivities to be proposed.

\section{FBG FABRICATION PROCESS}

2.1 Optical fibres and pre-processing preparation

The investigation was carried out using Ge-B-F doped photo-sensitive fibres (Fibercore PS1250). The fibres are composed of a silica cladding and a core of $5 \%$ wt germania with boron phosphorus doped-silica. They are single-mode fibres with a polyacrylate coating. The fibre has a cladding diameter of $125 \mu \mathrm{m}$ and a cut-off wavelength of $1250 \mathrm{~nm}$. The polyacrylate coating is highly absorbing in the ultra violet (UV) region of the spectrum, requiring removal of the coating prior to fabrication of the FBG. A $20 \mathrm{~mm}$ long section of the polyacrylate buffer coating was removed from the fibre surface before the inscription of FBGs. The process of removing the coating reduces the strength of the optical fibres to some extent, depending on the stripping method employed. In a previous investigation [6], a chemical-solvent stripping method was determined to have the least damaging effect, maintaining $80 \%$ of the strength of the pristine optical fibre. Therefore, this method was employed in this investigation. The fibres were soaked in the chemical solvent, and a lint-free tissue soaked with acetone was used to remove any coating residue.

Seven sets of fibres, each set comprising 15 fibre samples of a length of 300mm, were prepared under the same conditions in order to determine the Weibull distribution of the fibre strength. The details of the sets of fibres and the processing parameters are listed in Table 1. 
Among all the sets, only set D was subjected to hydrogen loading prior to the coating removal and UV-irradiation. The fibres were placed in a steel chamber filled with hydrogen at a pressure of 120 bar. The hydrogenation took place for one week at room temperature.

\subsection{UV-irradiation process}

The fabrication of FBGs was accomplished by exposing the stripped section of the fibre to UVbeams in the phase mask configuration [12]. The exposure parameters (the wavelength of light, pulse energy and total exposure dose) listed in Table 1 were different for each set of fibres,. Three lasers were used with wavelengths of $246 \mathrm{~nm}, 255 \mathrm{~nm}$ and $266 \mathrm{~nm}$. The specifications of the lasers are detailed in Table 2. Samples of the fibre that were not hydrogen-loaded were irradiated at the three laser wavelengths, while the $255 \mathrm{~nm}$ irradiation was carried out on both the hydrogen and non-hydrogen loaded fibres to compare the difference of the FBG mechanical strength. The FBGs fabricated using the $245 \mathrm{~nm}$ laser had the Bragg wavelength of $1300 \mathrm{~nm}$ determined by the period of the phase mask used, and the Bragg wavelength was $1320 \mathrm{~nm}$ for the FBGs inscribed using the 255 and $266 \mathrm{~nm}$ lasers. The exposure time for each fibre was approximately 60s.

The reflectivity of the FBGs formed in the Ge-B-F doped fibre without $\mathrm{H}_{2}$-loading was in the range 23-30\%, while the FBGs formed in the hydrogenated fibres had a reflectivity of approximately $60 \%$.

After being processed, the fibres were kept in a desiccator $\left(23^{\circ} \mathrm{C}, 26.5 \% \mathrm{RH}\right)$ to protect the fibres from the effects of humidity, which has been shown previously to degrade the mechanical strength of optical fibres [13]. The time interval between the FBG fabrication and the mechanical testing was 7 days. 
2.3 Tensile tests and microscopic observations

Tensile tests were conducted to evaluate the mechanical strength of the fibre sets listed in Table 1. In the tensile test, the fibres were end-tabbed using cardboard tabs attached with a fast curing adhesive, to protect samples from failure within the grips of the tensile testing machine. The gauge length of the samples was $160 \mathrm{~mm}$ while the total length of the fibre was $260 \mathrm{~mm}$. The tensile tests were performed on an Instron Model 6025 tensile testing machine with a load cell of $100 \mathrm{~N}$ capacity. The tensile failure load was measured under a strain rate of $0.035 / \mathrm{min}$.

In each set of fibres, 15 specimen were tested. Tests were considered valid when fibres fractured within the gauge length. In practice it was observed that all of the fibres that had had their coating removed fractured within the stripped section and that those which had also been UV irradiated failed within the UV-exposed region. The tensile strength was then determined by dividing the failure load by the area of fibre glass (assuming a constant diameter of $125 \mu \mathrm{m}$ ). The test environment was maintained at $23^{\circ} \mathrm{C}$, and $55 \% \mathrm{RH}$. A two-parameter Weibull model was employed to analyse the strength distribution of each set of data. The Weibull modulus, $\mathrm{m}$, is defined by:

$\ln (-\ln (1-F(\sigma)))=m \ln \sigma-m \ln \bar{\sigma}$

Where $F(\sigma)$ is the cumulative failure probability at each stress level, $\sigma$. The parameter, $\mathrm{m}$, defines the distribution scale of each set of measured data. $\bar{\sigma}$ is the strength at which $63.3 \%$ of samples fail within a set of fibres.

When a fibre broke into two pieces during the tensile measurement, the two fracture surfaces were collected for Scanning Electron Microscopy (SEM) observation. The fracture morphology was used for analysing fracture mechanisms. Optical fibres that failed at high stresses were observed to shatter into small pieces so that fracture surfaces suitable for examination were not obtained. 


\section{RESULTS}

The tensile test results which were analysed using the two-parameter Weibull model are summarised in Table 3, showing the mean strength and the Weibull modulus. The mean strength of the as-received fibres, with coating intact, was as high as $4.95 \mathrm{GPa}$. Following removal of the polyacrylate coating, the mean strength was reduced to $3.38 \mathrm{GPa}$. The mechanical strength was significantly reduced by the process of removing the coating. The width of the strength distribution increased, with a corresponding decrease of the m-value from 66 to 3.1 as seen in Fig. 1 and Table 3. Two previous investigations $[4,14]$ revealed no strength degradation when hot sulphuric acid was used to strip the coating. However, the mechanical measurements were conducted immediately after the stripping process. It has been found that the time lapse between the acid-stripping process and strength measurement has a major impact on the strength of stripped fibres [6]. However the fibres stripped chemically by the method used in this investigation showed little sensitivity to the time lapse under a similar storage condition following 7 day storage in a desiccator. The chemically-stripped fibres retained $68 \%$ of their original strength, compared with a $50 \%$ strength loss by using the acid-stripping method. In contrast, measurements on Corning SMF 28 fibres following chemical-stripping process showed a significant degradation with a mean strength of 0.92 GPa [8]. Hence, a large variation in the strength of stripped fibres may exist depending on what stripping method is used and how carefully the fibres are handled.

Stripped fibres that were subsequently UV-irradiated show a further reduction in the mean strength and a slight decrease in m-values. However the extent of UV degradation depends on the wavelength of the UV irradiation, and whether or not the fibre had been hydrogen-loaded, as seen in Table 3.

3.1 Influence of UV-parameters on the FBG mechanical strength 
Figure 1 shows the failure stress of irradiated PS1250 fibres plotted against the cumulative failure probability, comparing the strength of FBGs without hydrogen loading formed under irradiation at the different wavelengths. The data points show scatter and are non-linear on the Weibull plot for the UV-exposed fibres. If only one mechanism is responsible for the fracture of a material, the Weibull plot is expected to show a linear dependence of the failure stress on cumulative failure probability, as is seen in the as-received optical fibres (Set A) in Fig. 1. In the as-received fibres, a polymer coating is applied just after the fibres are drawn, which ensures that no mechanical defects are introduced. In the process of FBG fabrication, defects of various sizes could be induced during the coating removal and UV-irradiation, producing the wide distribution of the strength and nonlinear performance.

a) Wavelength vs. mechanical strength

When plotting the mean tensile strength against the wavelength of the lasers which were used to fabricate the FBGs, an almost linear relationship is obtained as shown in Fig. 2. Exposure at shorter UV wavelengths appears to produce FBGs with greater mechanical strength (Set E) when compared to that obtained using the longer UV wavelengths (Set F). FBG fabrication at $246 \mathrm{~nm}$ (Set E) gives the FBGs a higher mean strength than those exposed at 255 (Set C) and 266nm (Set F). The total energy dose of the 255nm exposure (Set C) is more than 39 times that of $266 \mathrm{~nm}$ exposure (Set F), but the strength of Set $\mathrm{C}$ fibres is 1.8 times higher than that of Set F fibres. It appears that the total exposure energy dose has little influence on the mechanical resistance while the laser wavelength produces a significant effect. When Set F is compared to Set G, both of which were irradiated by the $266 \mathrm{~nm}$ laser, Set F shows only 10\% higher strength than Set G, even though the total exposure dose for the former set is $28 \%$ of the latter. Feced et al [8] reported a similar result where a higher strength was achieved using the laser operating at $193 \mathrm{~nm}$ than that obtained using UV irradiation at $248 \mathrm{~nm}$ for a pulse fluence of $1 \mathrm{~J} / \mathrm{cm}^{2}$. The FBGs had strengths of $0.84 \mathrm{GPa}$ and $0.69 \mathrm{GPa}$, 
respectively. The FBGs fabricated using the three wavelength lasers in this investigation show higher strength than those reported in [8]. This could be because that the stripped fibres exhibited a strength of $0.92 \mathrm{GPa}$ prior to irradiation, significantly lower than that of the stripped fibres in the present investigation (3.38 GPa).

b) UV pulse peak intensity vs. mechanical strength

The influence of peak pulse intensity on the mechanical strength is shown in Figure 3. Although the fibre sets were fabricated using different laser wavelengths, the general trend shows that an increase of pulse peak intensity reduces the mechanical strength. The exception is Set E which shows a higher strength than that of Set C though Set E was fabricated using a higher peak pulse energy. The poor mechanical resistance of FBGs fabricated at 266nm could be caused by both the longer laser wavelength and the high pulse energy which is 3.5 times that of the $246 \mathrm{~nm}$ lasers and over 80 times that of the copper vapour system $(255 \mathrm{~nm})$. Hence it is difficult to distinguish between these two variables on the basis of the present results. However, the peak energy of a single pulse seems less influential than the laser wavelength in reducing the strength. Set E, fabricated using a $246 \mathrm{~nm}$ laser, has a greater strength than Set C, fabricated using $255 \mathrm{~nm}$ in spite of the pulse peak intensity of the former laser being 20 times higher than that of the latter. This suggests that laser wavelength has a more profound influence on mechanical strength than does the pulse peak intensity.

\subsection{Influence of hydrogen loading on the FBG mechanical strength}

As shown in Table 3, UV-irradiation under similar conditions induced a strength degradation of $36 \%$ for fibre Set C and 70\% reduction for fibre Set D, which contained the same type of fibres but Set D had been hydrogen-loaded. To show the influence of hydrogen loading, the failure stress of the irradiated fibres with and without hydrogen loading is plotted against cumulative failure 
probability in Fig. 4. It is clear that hydrogen loading had a significant impact on mechanical strength, the mean strength being less than $50 \%$ of that of the fibres that had not been hydrogen loaded. The power density and total energy dose used during the exposure of Set D, the hydrogenloaded fibres, were lower than those applied to the Set C (not hydrogen-loaded), as seen in Table 3.

Limberger et al [11] investigated the mechanical strength of Ge-doped photosensitive fibres both with and without hydrogen-loading prior to UV-irradiation. Their results revealed a small degradation of the mechanical strength, from $4.74 \mathrm{GPa}$ to $4.62 \mathrm{GPa}$, along with a decrease in the mvalue from 165 to 69 , for fibres that were processed in $\mathrm{H}_{2}$ at 120 Bar for 16 months. However, no degradation was observed when the fibre was loaded in $\mathrm{H}_{2}$ for two weeks. The short-term $\mathrm{H}_{2}{ }^{-}$ loading process causes no reduction in the mechanical resistance of the fibre. However, this investigation shows that the mechanical strength of $\mathrm{H}_{2}$-loaded fibres is significantly reduced following UV-irradiation.

\section{Mechanical strength analysis from fracture surface observed by SEM}

The fibre fracture surfaces were observed under the SEM. If the optical fibres broke at a low stress ( $<1500 \mathrm{MPa}$ ), the fracture surfaces commonly showed a region of hackles radiating from a semicircular region as seen in Fig. 5. This semicircular region consists of a mist zone and a mirror zone where a flat surface is seen. The radius of the mirror zone varied with the measured failure stress. High strength fibres showed a small mirror zone. When the failure stress is between 1500 to $3000 \mathrm{MPa}$, the fracture morphology changed to a relatively flat surface without clear signs of a single crack initiation point, as shown in Fig. 6. No mirror zone is observed in the fracture surface in this case. The fracture surface shows a typical feature of a brittle material. When the failure stress was above $3000 \mathrm{MPa}$, the fibres were shattered into small pieces and could not be examined. The 
features of fracture morphology are related to the level of failure stress only, regardless of the treatment conditions of optical fibres.

An empirical relationship has been proposed by some investigators $[15,16]$ to correlate the mirror zone radius to the strength of glass fibres as:

$\sigma_{\mathrm{f}} \mathrm{r}^{1 / 2}=\mathrm{C}$

where: $\sigma_{\mathrm{f}}$ is the fracture stress of the fibre, $\mathrm{r}$ is the radial depth of the mirror zone, and $\mathrm{C}$ is a constant

A linear fit is expected when plotting $\sigma_{\mathrm{f}}$ against $\left(\mathrm{r}^{1 / 2}\right)^{-1}$. Levengood's work [16] reported a linear relationship between the depth of the mirror zone and the actual depth of the surface defect. The term $\left(\mathrm{r}^{1 / 2}\right)^{-1}$, derived from the depth of mirror zone measured in $\mathrm{H}_{2}$-loaded fibres, is plotted against strength in Fig. 7. The data points are located around a line. The linear regression fit to this line is obtained with a correlation coefficient of 0.85 .

SEM observations showed that 13 of the 14 failed specimens of the irradiated hydrogenated fibres (Set D) had a surface defect initiated fracture morphology. As seen in Fig. 4, apart from one fibre, all the failure stresses of hydrogenated fibres are below $1400 \mathrm{MPa}$. The exception exhibited a high failure strength of $1540 \mathrm{MPa}$, showing no sign of surface defect initiated failure. From this, it is evident that surface defects are a dominant factor controlling fracture in UV irradiated hydrogenated fibres. 
In contrast, in the set of UV irradiated non-hydrogenated fibres (set C), only three out of 14 samples showed signs that failure was initiated from the surface. These three fibres also had a failure strength of less than $1400 \mathrm{MPa}$. The fracture surface shown in Figure 6 exhibits the features that are seen in most of the fractured non-hydrogen loaded fibres with a failure stress above $1400 \mathrm{MPa}$.

\section{Examination of UV-induced strength degradation using fracture mechanics}

The SEM investigation revealed that the majority of the hydrogen-loaded fibres and some of the non-hydrogen-loaded fibres inscribed with FBGs had fractures originating at the surface from a defect of a size that is too small to be resolved in the SEM. AFM observations, reported in [17], have shown that the writing of FBGs is accompanied by creation of surface corrugations on the fibre, oriented perpendicular to the axis of the fibre and with a period equal to $455 \mathrm{~nm}$ for the FBGs written using a phase mask with a period of $900 \mathrm{~nm}$. Figure 8 shows a typical AFM image of the irradiated surface of a fibre inscribed with FBGs. The location of the surface troughs corresponds to the location of the peak of the intensity of the interference fringes. It was found that the depth of the corrugation increased with UV intensity and was significantly increased by hydrogen loading the fibres prior to FBG writing. It is conceivable that the corrugation depth may be related to size of defects promoting failure in the fibre tensile tests.

The relationship between fracture stress and defect size is governed by fracture mechanics. The fracture behaviour of glass fibres was originally investigated by Griffith [18], and his conclusions on the role of defect and fracture energies have been subsumed into modern fracture mechanics. Assuming that the surface defects may be considered to be cracks, the relationship between fracture stress and defect size is given by 
$\sigma_{f}=\frac{K_{I C}}{\beta \sqrt{\pi a}}$

where:

$\sigma_{\mathrm{f}}$ is fracture stress of the fibre

$\mathrm{K}_{\mathrm{IC}}$ is a material constant, known as the fracture toughness of the material

$\mathrm{a}$ is the defect depth

and $\beta$ is a geometrical constant

The surface troughs induced by UV-exposure may approximate to surface cracks. Thus the defect depth in Eqn 3 is taken from the measured surface trough depth based on AFM observation. Equation 3 suggests that plotting fracture stress against inverse square root of defect depth should yield a straight line. Fig 9 shows a plot of mean failure stress against the defect depth of fibres from sets B, F and G. The $0.7 \mathrm{~nm}$ defect depth for Set B that was not UV-irradiated was the amplitude value of the AFM measured surface roughness. The corrugation depth for Set $\mathrm{F}$ was $1.7 \mathrm{~nm}$ measured from the AFM observation in the centre of irradiated region where the UV intensity is known to be the highest. The depth for Set $G$ was $6.1 \mathrm{~nm}$, calculated from the relationship of the corrugation amplitude and UV pulse intensity derived from the measured data in [17]. A linear line is obtained, suggesting that the changes in corrugation introduced by UV irradiation cause the reduction of fracture stress.

More quantitative assessment can be made by assuming that the value of $\beta$ is 1.0 , representing a semi-elliptical crack configuration [19] and that the fracture toughness of glass fibres is 0.8 MPa.m ${ }^{1 / 2}$ [20]. In Eqn 3, these values are then used to calculate the fracture stresses $\sigma_{f}$, with the measured corrugation depth as the defect depth. The result, also plotted in Fig. 9, shows that the calculated fracture stresses are considerably overestimated by a factor of between 5 and 9 . There is 
high confidence in the values of fracture toughness used and the value of the constant $\beta$ for this geometry configuration. Hence it is likely that the errors lies in an under estimation of the defect sizes.

Values of defect depths necessary to produce the measured fracture stresses can be calculated using the same expression (Eqn 3) by putting the measured fracture stresses into the equation lie between 18 and $170 \mathrm{~nm}$, which is approximately 25-80 times greater than the measured corrugation depth. The discrepancy could arise from the fact that only a small area $\left(10 \times 10 \mu \mathrm{m}^{2}\right)$ in UV irradiated area was observed using AFM. More severe defects present in the rest of the irradiated area will not be detected. The value of the corrugation depth used in the calculations and in Figure 9 is a mean value across a $10 \mu \mathrm{m}$ width, which is unlikely to represent the deepest defect that will initiate the failure. The UV beam profile is not completely uniform resulting in local "hot spots” which may introduce more severe local damage. These local damage sites will become the defect initiating brittle failure.

Secondly it is possible that the defect opening is so fine that the AFM cannot measure the true depth, only the surface relief. In the FBG fabrication process, the high intensity UV interference pattern creates the surface corrugation consisting of a series of compacted troughs. The process may also produce cracks or defects which extend from the points of greatest corrugation depth into the fibre. The driving force for the cracks could be local thermal stresses, generated by the alternating bands of high and low temperature which the interference pattern will produce within the fibre. It may also be due to the existence of stress concentration between the irradiated and non-irradiated bands. 
Despite the discrepancy in the measured corrugation depth and the calculated defect sizes necessary to produce the observed failure stresses, changes in corrugation depth appear to represent the changes in real defect size in their effect on fracture stress. Increased pulse power intensity and hydrogen loading of the fibres are observed to cause increased corrugation depth [17] and thus decreased mean fracture stress.

It seems likely that at the high intensity regions of the interference pattern, a process of photo ablation could take place, locally removing the surface material along the interference fringe. Thus the observed effect of increased laser intensity may be explained, which will increase corrugation depth and decrease fracture stress. There may exist thermal stresses generated across the fringes which could aid a crack nucleation process. However, whether the effect of different laser wavelengths on the change of fracture stresses is linked to the change of corrugation depth is unclear, because the corrugation depth was investigated using the $266 \mathrm{~nm}$ laser only .

\section{CONCLUSIONS}

(1) Measurements of the tensile strength of batches of optical fibres containing FBGs produced using laser wavelengths of 246, 255 and $266 \mathrm{~nm}$ and different peak intensities have demonstrated that:

(a) Mean fibre tensile strength is reduced from $3.38 \mathrm{GPa}$ in the un-irradiated condition to a maximum of $2.52 \mathrm{GPa}$ after gratings are written.

(b) Further decreases in mean strength to as low as $1.0 \mathrm{GPa}$ are produced by increases in laser peak intensity, and increases in wavelength from 246 to $266 \mathrm{~nm}$. 
(c) Hydrogen loading the fibres prior to grating inscription, causes reduction in peak tensile strength from 2.16 GPa to 1.01 GPa for the same fabrication condition using the $255 \mathrm{~nm}$ laser

(2) Fracture surface observation using the SEM have revealed that fibres failing at stresses less than $1500 \mathrm{MPa}$ showed fractures indicating a single defect origin from the surface.

(3) Atomic Force Microscopy (AFM) of fibre surfaces after irradiation showed the presence of surface corrugations with a period identical to that of the interference pattern used to form the FBG.

(4) A relation between inverse square root of corrugation depth and mean fracture stress has been found, suggesting a fracture mechanics basis for the influence of the surface corrugation on mean tensile strength

(5) Defect depths to produce observed strength are calculated to be between 25-80 times greater than the observed corrugation depths. It is concluded that the mean corrugation depths represent a population of defects whose extremes are the defects which cause failure.

\section{ACKNOWLEDGMENTS}

The authors would like to acknowledge AEA Technology Plc and the Civil Aviation Authority, UK for funding this project. Special appreciation goes to Dr K H Winters, Dr R Davidson and Prof J Bristow for their collaboration and valuable discussions. The authors are grateful to Oxford Lasers for access to the copper vapour laser system.

\section{REFERENCES}


1. R. Kashyap, Fibre Bragg Gratings, Academic Press, 1999, p. 13-35.

2. B. Malo, J. Albert, K. O. Hill, F. Bilodeau and D. C. Johnson, Electron., Lett. 30(1994), P.442444

3. P. J. Lemaire, R. M. Atkins, V. Mizrahi and W. A. Reed, Elctron Lett., 29 (1993), p.1191-1193.

4. D. Varelas, D. M. Costantini et al, Optics Letters, 23(1998), p. 397-399.

5. L. Dong, J. L. Archambault, L. Reekie, P. St. J. Russell and D. N. Payne, Electron. Lett. 29 (1993), p.1577-1578.

6. C. Y. Wei, S. W. James, C. C. Ye, R. P. Tatam and P. E. Irving, The $6^{\text {th }}$ Annual International Symposium on Smart Materials and Structures, SPIE vol. 3670 (1999), p. 164-170.

7. D. Varelas, H. G. Limberger, R. P. Salathe and C. Kotrotsios, Electron. Lett., 33(1997), p. 804806.

8. R. Feced, M. P. RoeEdwards, S. E. Kanellopoulos, N. H. Taylor and V. A. Handerek, Electron Lett, 33 (1997), pp. 157-159.

9. C. G. Askins, M. A. Putnam, H. J. Patrick, and E. J. Friebele, Electron. Lett., 33 (1997), p. 1333-1334.

10. R. M. Atkins, P. J. Lemaire, T. Erdogna and V. Mizrahi, Electron. Lett, 29(1993), P. 1234-1235.

11. H. G. Limberger and D. Varelas, Reliability of Optical Fibres and Components, Final Report of COST 246, Ed. by Volotinen, Griffioen, Gadonna and Limberger, Springer, 1999, p.351-352.

12. M. L. Dockney, S. W. James and R. P. Tatam, Meas. Sci. Technol., 7 (1996), P. 445-448.

13. S. L. Semijonov, M. M. Bubnov and O. V. Khleskova, Optical Network Engineering and Integrity, SPIE, vol 2611 (1996), P. 49-54.

14. M. J. Matthewson, C. R. Kurkjian and J. R. Hamblin, Journal of Lightwave Technology, 15 (1997), p. 490-497.

15. A. C. Jaras, B. J. Norman and S. C. Simmens, Journal of Materials Science, 18 (1983), p.24592465. 
16. W. C. Levengood, Journal of Applied Physics, 29(1958), p.820-826.

17. C. Y. Wei, C. C. Ye, S. W. James, R. P. Tatam and P. E. Irving, AFM observation on surface topography of fibre Bragg grating fabricated on germania-boron codoped fibres and hydrogenloaded fibre (submitted to Optical Materials).

18. A. A. Griffith, Phil. Trans. Roy. Soc. A221(1920), p.163.

19. D. P. Rooke and D. J. Cartwright, Compendium of Stress Intensity Factors, London, 1976, p.297-298.

20. W. Griffioen, T. Svensson and B. Friderich, the Proceedings of the $43^{\text {rd }}$ International Wire and Cable Symposium, Georgia, 1994, p. 750-757 


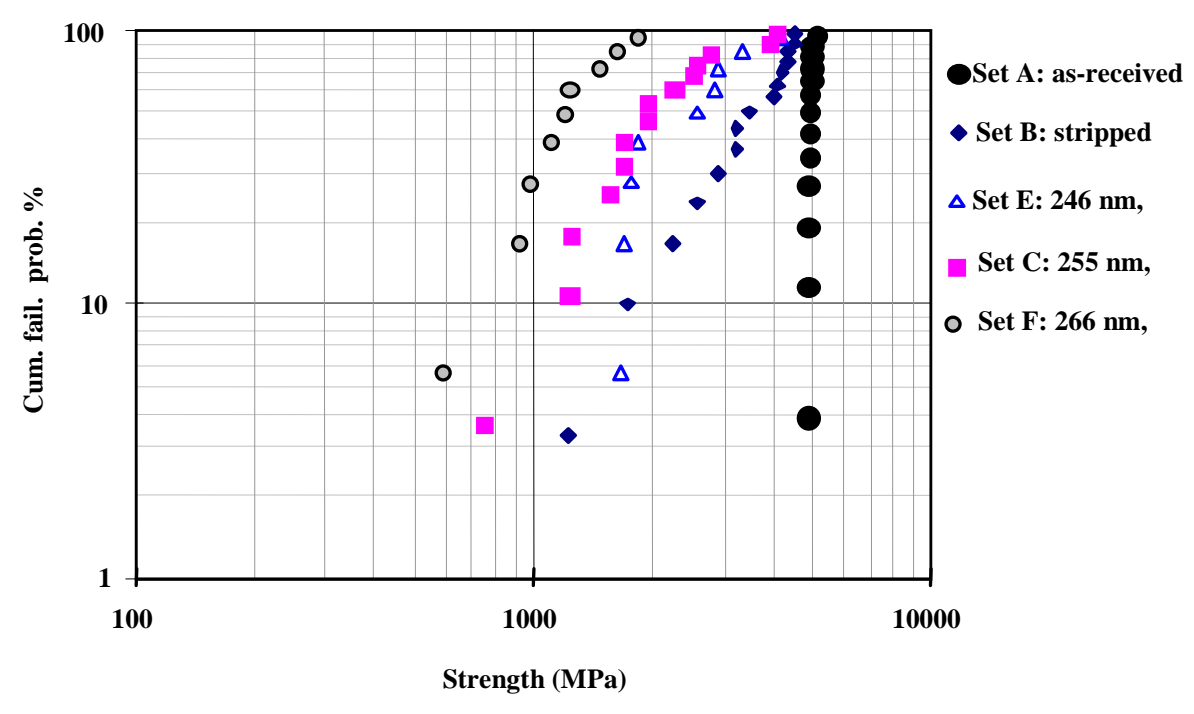

Fig. 1 Weibull plot of fibre strength for Ge-B-F doped fibres in the form of as-received, coatingremoved and irradiated under different UV-wavelengths

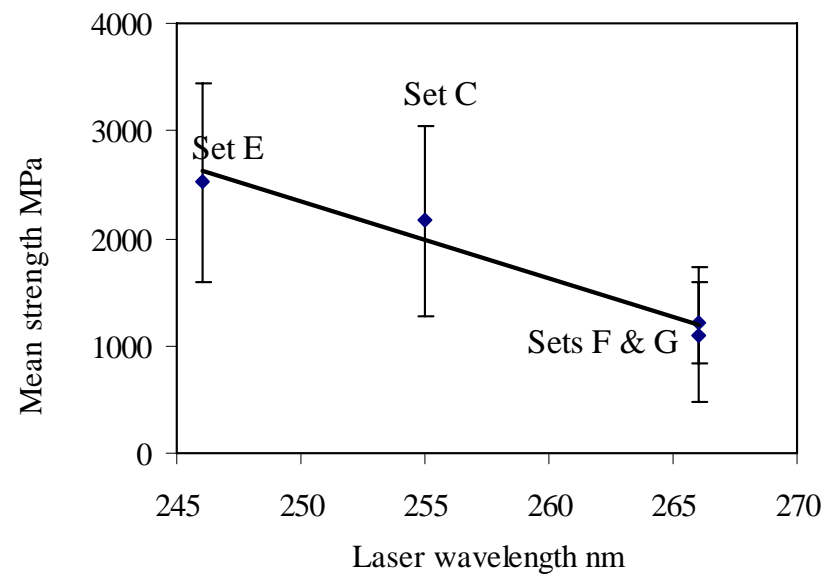

Fig. 2 Mechanical strength of FBGs fabricated at different laser wavelengths

Solid line: fitted trendline 


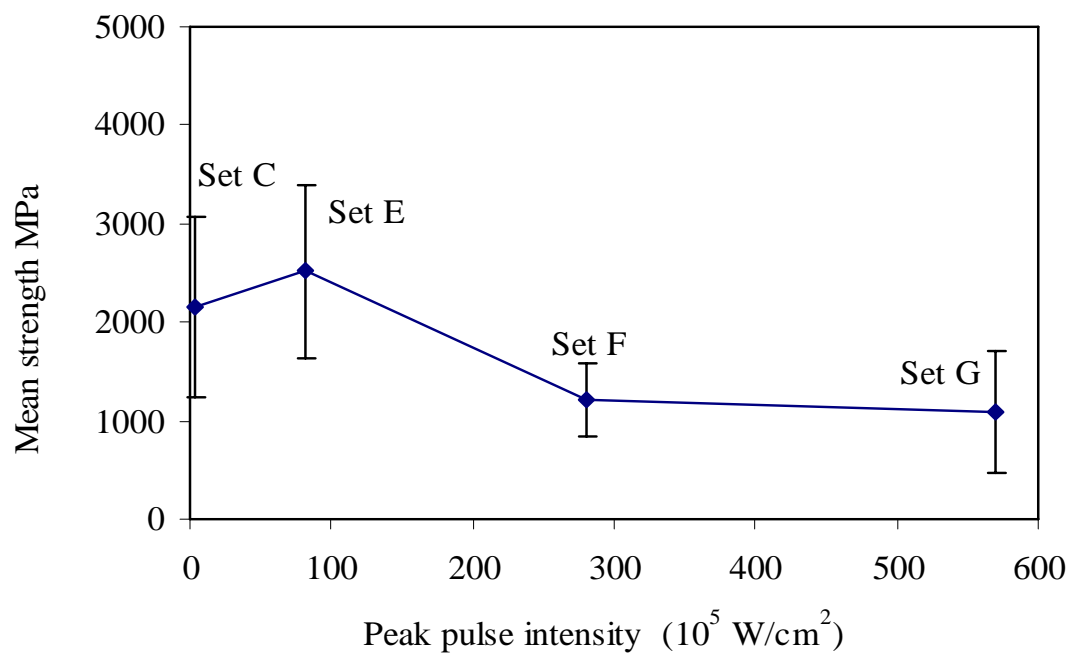

Fig. 3 Mechanical strength of FBGs fabricated in Ge-B codoped fibres using different peak pulse intensity

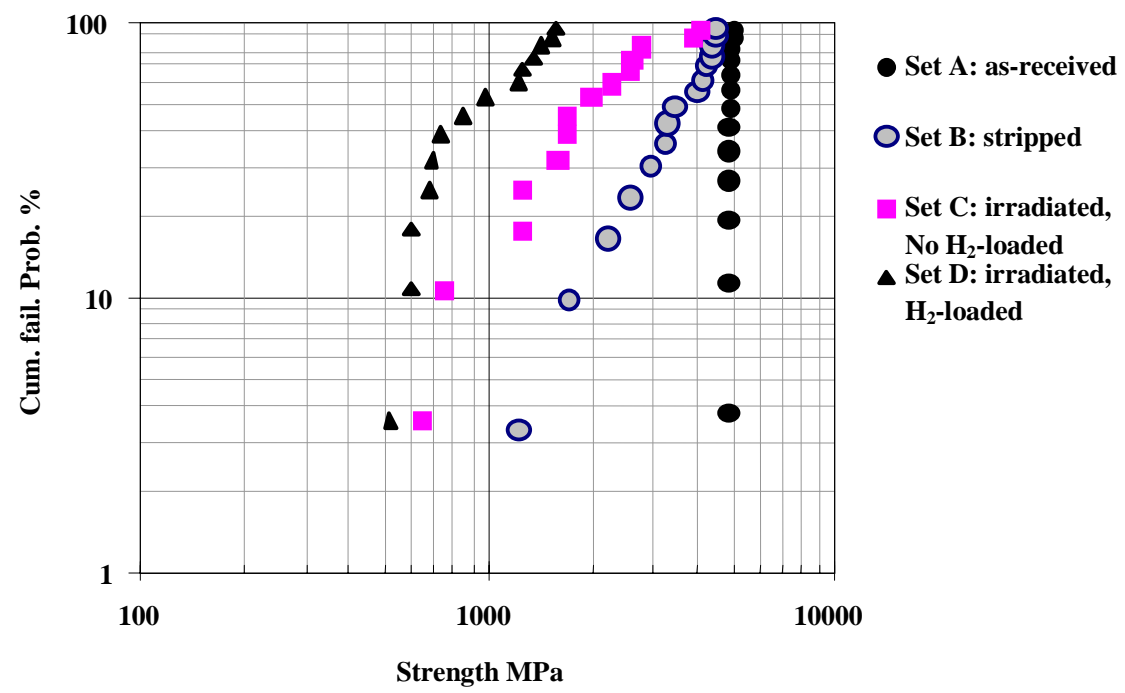

Fig. 4 Weibull plot of fibre strength for irradiated Ge-B codoped fibres with/without hydrogen loading (exposed to UV radiation at $255 \mathrm{~nm}$ ) 


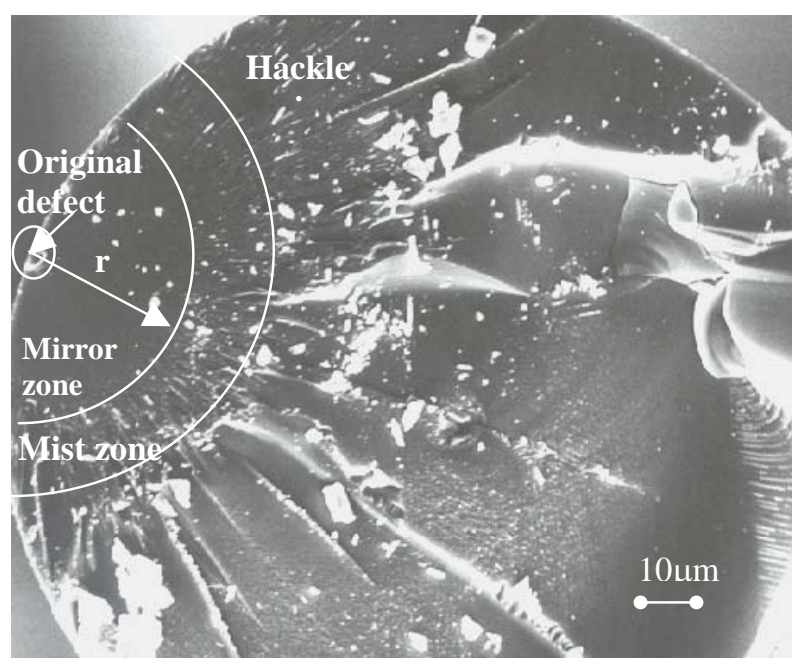

Fig. 5 Fracture surface showing failure initiated from surface, a mirror zone, mist zone and hackle region in a hydrogen-loaded and UV irradiated fibre with a fracture stress of $603 \mathrm{MPa}$.

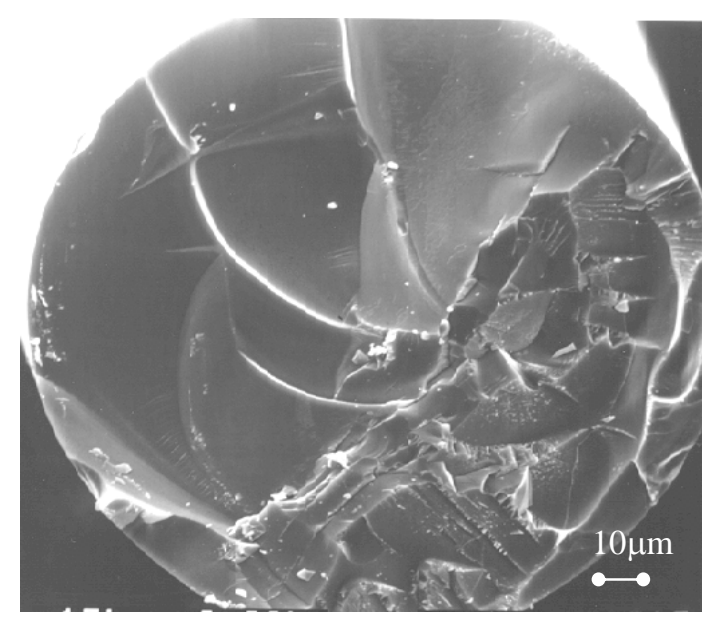

Fig. 6 Fracture surface without signs of surface crack initiation observed in a non-hydrogen loaded and irradiated fibre with a fracture stress of $1688 \mathrm{MPa}$ 


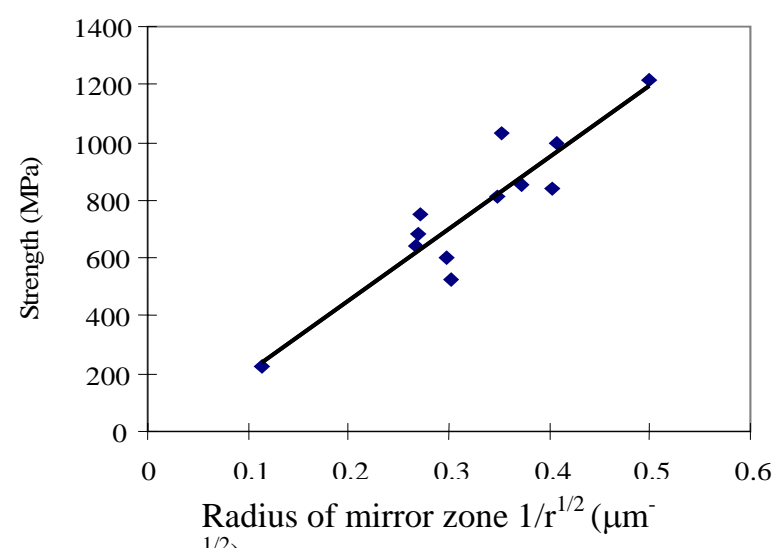

Fig.7 Relation between strength and the depth of the mirror zone in the hydrogen-loaded and UV irradiated optical fibres.

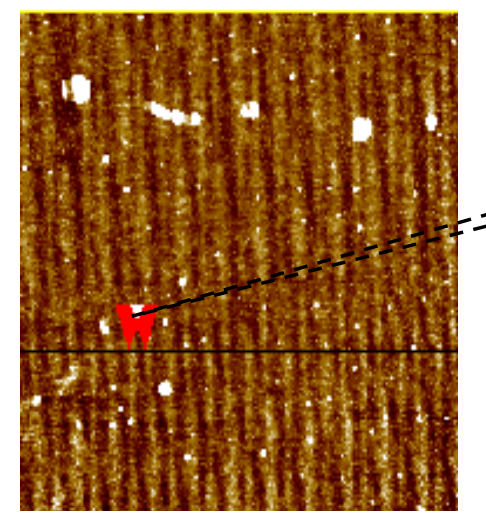

a) 2-D surface $(10 \times 10 \mu \mathrm{m})$

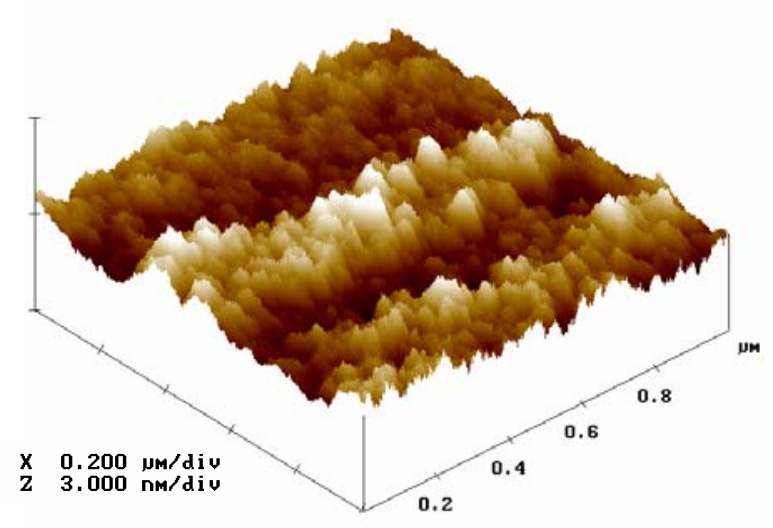

c) 3-D surface $(1 \times 1 \mu \mathrm{m})$

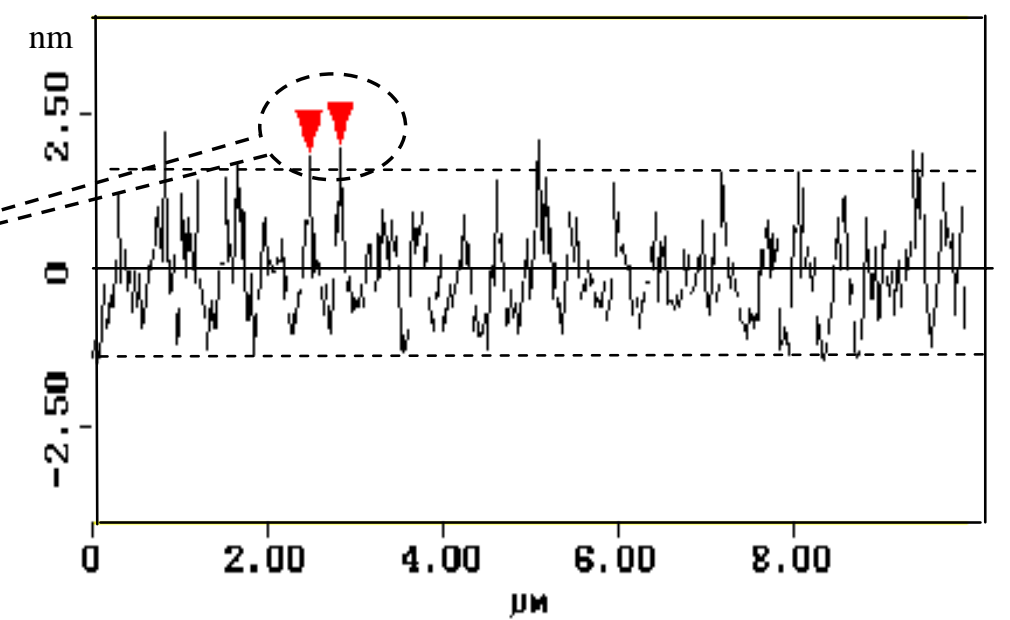

b) Surface roughness spectrum

Fig. 8 AFM image of irradiated Ge-B codoped fibre surface (FBG inscription condition: 266nm laser, $2.8 \mathrm{~W} / \mathrm{cm}^{2}$ pulse average density) 


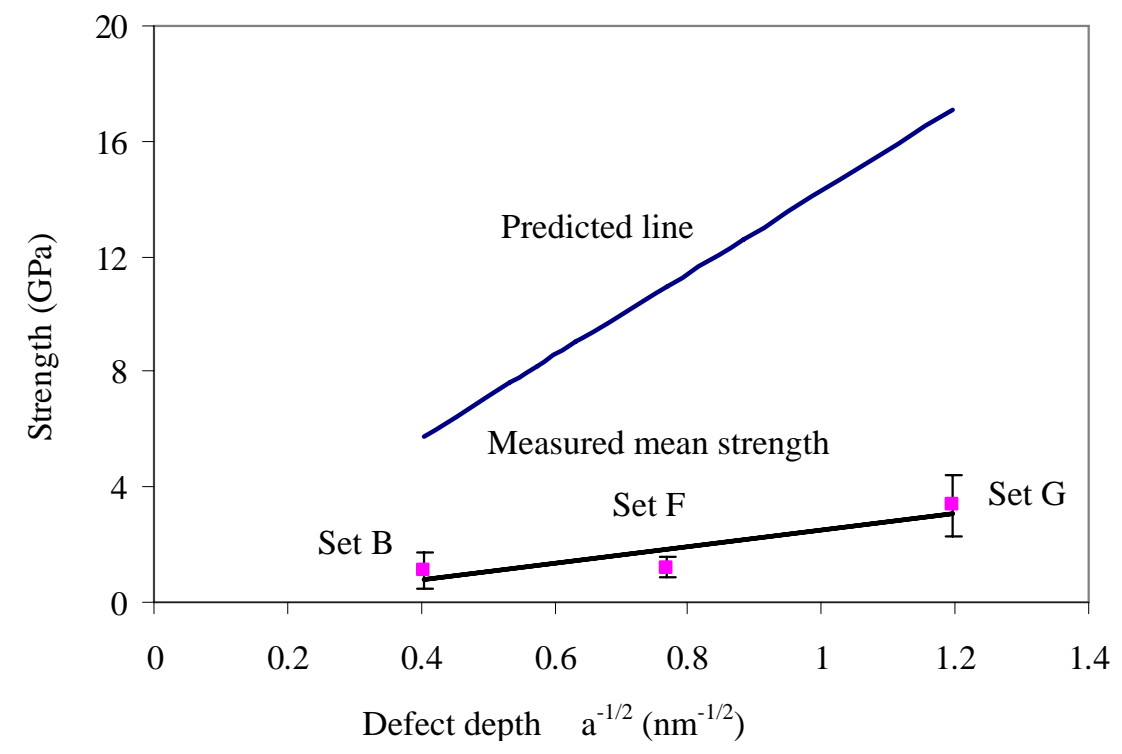

Fig. 9 Relation between strength and surface defect depth of optical fibres 
Table 1: Process parameters of optical fibres

\begin{tabular}{|l|l|l|c|l|c|}
\hline $\begin{array}{l}\text { Fibre } \\
\text { set }\end{array}$ & Fibre type & \multicolumn{4}{|c|}{ UV exposure parameters } \\
\hline & $\lambda(\mathrm{nm})$ & $\begin{array}{l}\text { Pulse average } \\
\text { intensity }\left(\mathrm{W} / \mathrm{cm}^{2}\right)\end{array}$ & $\begin{array}{l}\text { Pulse peak } \\
\text { intensity }\left(\mathrm{W} / \mathrm{cm}^{2}\right)\end{array}$ & $\begin{array}{l}\text { Total dose } \\
\left(\mathrm{J} / \mathrm{cm}^{2}\right)\end{array}$ \\
\hline A & As-received & \multicolumn{5}{|c|}{} \\
\hline B & Stripped & & 74 & $3.5 \times 10^{5}$ & 6640 \\
\hline C & Irradiated & 255 & 55 & $2.6 \times 10^{5}$ & 4914 \\
\hline D & $\begin{array}{l}\text { H2-loaded \& } \\
\text { Irradiated }\end{array}$ & 255 & 2.0 & $8.1 \times 10^{6}$ & 180 \\
\hline E & Irradiated & 246 & 1.4 & $2.8 \times 10^{7}$ & 170 \\
\hline F & Irradiated & 266 & 4.5 & $5.7 \times 10^{7}$ & 610 \\
\hline G & Irradiated & 266 & & & \\
\hline
\end{tabular}

Table 2: Laser parameters

\begin{tabular}{|l|c|c|c|c|}
\hline Laser type & $\begin{array}{l}\text { Laser } \\
\text { wavelength }\end{array}$ & $\begin{array}{l}\text { Pulse width } \\
\text { (ns) }\end{array}$ & $\begin{array}{l}\text { Pulse energy } \\
\text { (mJ) }\end{array}$ & Repetition rate \\
\hline $\begin{array}{l}\text { Injection seeded, frequency } \\
\text { doubled Nd:YAG pumped } \\
\text { dye laser with frequency } \\
\text { doubled sum-frequency } \\
\text { mixed output }\end{array}$ & 246 & 10 & $\leq 3$ & $25 \mathrm{~Hz}$ \\
\hline $\begin{array}{l}\text { Frequency-doubled copper } \\
\text { vapour laser }\end{array}$ & 255 & 35 & 0.2 & $6 \mathrm{kHz}$ \\
\hline $\begin{array}{l}\text { Injection seeded, } \\
\text { frequency-quadrupled } \\
\text { Nd:YAG laser }\end{array}$ & 266 & 5 & $\leq 150$ & $10 \mathrm{~Hz}$ \\
\hline
\end{tabular}

Table 3: Mechanical strength and Weibull modulus of optical fibres and FBGs

\begin{tabular}{|l|l|l|l|l|l|l|l|}
\hline Fibre set & A & B & C & D & E & F & G \\
\hline $\begin{array}{l}\text { Mean } \\
\text { strength } \\
(\mathrm{GPa})\end{array}$ & 4.95 & 3.38 & 2.16 & 1.01 & 2.52 & 1.21 & 1.10 \\
\hline $\begin{array}{l}\text { Weibull } \\
\text { modulus, m }\end{array}$ & 66.6 & 3.1 & 2.2 & 2.4 & 3.2 & 3.6 & 1.2 \\
\hline
\end{tabular}

